These articles have been accepted for publication in the British Journal of Dermatology and are currently being edited and typeset. Readers should note that articles published below have been fully refereed, but have not been through the copy-editing and proof correction process. Wiley-Blackwell and the British Association of Dermatologists cannot be held responsible for errors or consequences arising from the use of information contained in these articles; nor do the views and opinions expressed necessarily reflect those of Wiley-Blackwell or the British Association of Dermatologists

This article is protected by copyright. All rights reserved.

Received Date : 08-Jun-2014

Revised Date : 14-Jul-2014

Accepted Date : 04-Aug-2014

Article type : Clinical and laboratory investigations

\title{
Identification of Loci Associated with Late-Onset Psoriasis Using Dense Genotyping of Immune-Related Regions
}

\author{
H.L. Hébert ${ }^{1,2}$, J. Bowes ${ }^{2}$, Rh.L.I. Smith ${ }^{2}$, E. Flynn ${ }^{2}$, R. Parslew ${ }^{3}$, A. Alsharqi ${ }^{3}$, \\ N.J. McHugh ${ }^{4}$, J.N.W.N. Barker ${ }^{5}$, C.E.M. Griffiths ${ }^{1}$, A. Barton ${ }^{2,6}$, R.B. Warren ${ }^{1}$ \\ ${ }^{1}$ The Dermatology Centre, Salford Royal NHS Foundation Trust, University of Manchester, \\ Manchester Academic Health Science Centre, Manchester M6 8HD, U.K. \\ ${ }^{2}$ Arthritis Research UK Centre for Genetics and Genomics, University of Manchester, Manchester \\ Academic Health Science Centre, Manchester, M13 9PT, U.K. \\ ${ }^{3}$ Department of Dermatology, Royal Liverpool and Broadgreen University Hospitals NHS Trust, \\ Liverpool, U.K. \\ ${ }^{4}$ Royal National Hospital for Rheumatic Diseases and Department Pharmacy and Pharmacology, \\ University of Bath, Bath, U.K. \\ ${ }^{5}$ Division of Genetics and Molecular Medicine, King's College London School of Medicine, King's \\ College London, London, SE1 9RT, U.K. \\ ${ }^{6}$ NIHR Manchester Musculoskeletal Biomedical Research Unit, Central Manchester Foundation Trust, \\ Manchester Academic Health Science Centre, Manchester, U.K.
}

Corresponding author: Dr Richard B. Warren

The Dermatology Centre, Salford Royal NHS Foundation Trust, University of Manchester, Manchester Academic Health Science Centre, Manchester M6 8HD, U.K.

Tel: 44 (0)161 2064344 
This article is protected by copyright. All rights reserved.

Fax: $44(0) 1612061095$

Email: richard.warren@manchester.ac.uk

Short title: A genome-wide association study of late-onset psoriasis

Abbreviations: $\mathrm{BP}$, base pairs; $\mathrm{Chr}$, chromosome; $\mathrm{Cl}$, confidence interval; $\mathrm{EOP}$, early-onset psoriasis; eQTL, expression quantitative trait locus; GEC, Genetic type 1 error calculator; GWAS, genome-wide association study; $H L A-C$, human leukocyte antigen-C; IFIH1, interferon induced with helicase $C$ domain 1; IL12B, interleukin-12B; IL1R1, interleukin-1 receptor, type 1; IL23A, interleukin-23A; IL23R, interleukin-23R; LC, Langerhans' cell; LD, linkage disequilibrium; LOP, late-onset psoriasis; MAF, minor allele frequency; OR, odds ratio; PCA, principal components analysis; SNP, single-nucleotide polymorphism; Th1, T-helper cell 1; Th17, T-helper cell 17;TRAF3IP2, tumour necrosis factor receptor-associated factor 3 interacting protein 2;ZNF313, zinc finger protein 313.

Funding Sources: H.L. Hébert is funded by a Ph.D. studentship from Abbott (now AbbVie); J. Bowes and A. Barton are funded by Arthritis Research UK (grant ref. 20385). C.E.M. Griffiths is funded in part by the Medical Research Council and the NIHR Manchester Biomedical Research Centre.

Conflict of Interest: R.B. Warren has acted as a consultant and/or speaker for AbbVie, AMGEN, Janssen Cilag, Leo Pharma, Pfizer, Schering Plough (now MSD) and Novartis; C.E.M. Griffiths has acted as consultant for and/or received research grants from AbbVie, Actelion, Biogen-IDEC, Biotest BMS, Centocor, Incyte, Janssen-Cilag, Leo Pharma, MSD, Novartis, Pfizer, Sandoz, Trident and UCB Pharma; J.N.W.N. Barker has acted as a consultant to Abbott, Janssen-Cilag, Novartis, ScheringPlough and Wyeth and has lectured at sponsored symposia for the above companies; A. Barton has acted as consultant for and/or received research grants from Abbott, Eli-Lilly and Pfizer.

\section{What's already known about this topic?}

- Chronic plaque psoriasis can be dichotomised into early-onset (onset $<40$ years) and lateonset (onset $\geq 40$ years) subtypes

- Genetic studies have so far focussed on early-onset psoriasis, identifying 36 loci in the Caucasian population

- Late-onset psoriasis has generally been neglected in genetic studies 
This article is protected by copyright. All rights reserved.

\title{
What does this study add?
}

- The largest and first genome wide study of a late-onset psoriasis cohort to date demonstrating a novel association at IL1R1, which is specific for late-onset psoriasis

- Significant association of eight loci previously identified in early-onset psoriasis demonstrating overlap between early and late onset psoriasis

\begin{abstract}
Background: Chronic plaque psoriasis can be sub-divided into two groups according to age of onset; type 1 (early-onset: before 40 years) and type 2 (late-onset: at or beyond 40 years). So far, 36 genetic loci have been associated with early-onset psoriasis in Caucasian genome-wide association studies, whilst few studies have investigated genetic susceptibility to late-onset psoriasis.
\end{abstract}

Objectives: The aim of the current study was to characterise the genetics underpinning late-onset psoriasis.

Methods: We genotyped 543 late-onset psoriasis cases and 4,373 healthy controls using the Immunochip array; a dense genotyping chip containing single nucleotide polymorphisms previously associated with autoimmune diseases. Imputation using SNP2HLA and stepwise logistic regression analysis was performed for markers spanning the HLA gene region.

Results: Two loci (HLA-C and IL12B) previously associated with early-onset psoriasis showed significant association at genome-wide threshold in the current study $\left(P<5 \times 10^{-8}\right)$. Six more loci (TRAF3IP2, IL23R, ZNF313, IFIH1, IL23A and HLA-A) showed study-wide significant association $\left(\mathrm{P}<2.3 \times 10^{-5}\right.$; calculated using Genetic type 1 error calculator). Additionally, we identified an association at IL1R1 on chromosome $2 q 13$, which is not associated with early-onset disease.

Conclusion: This is the largest study to date of genetic loci in late-onset psoriasis and demonstrates the overlap that exists with early-onset psoriasis, but also suggests that some loci are associated exclusively with late-onset psoriasis. 
This article is protected by copyright. All rights reserved.

\section{Introduction}

Chronic plaque psoriasis affects around $2 \%$ of the population and is characterised by a bimodal distribution in age of onset allowing the disease to be dichotomised into early-onset (EOP; type 1 psoriasis; onset before 40 years of age) and late-onset (LOP; type 2 psoriasis; onset at or beyond 40 years of age $)^{1 ; 2}$. The majority of chronic plaque psoriasis patients present as EOP cases ( 75\%).

A genetic component to the disease is well documented with a recent meta-analysis of three genome-wide association studies (GWAS) increasing the number of loci associated with psoriasis to $36^{3}$. The pathways implicated in these studies include antigen presentation (HLA-B/C, ERAP1); Thelper cell 1 (Th1) and T-helper cell 17 (Th17) signalling (IL12B, IL23R, ZNF313); NFKB and TNF- $\alpha$ pathways (TRAF3IP2, TNFAIP3, TNIP1, TYK2) and skin barrier function (LCE). However, large-scale genetic studies have focused mainly on EOP, with few or even no LOP patients contained in the cohorts studied. As a consequence, genetic studies in LOP are lacking. The heritability of EOP appears greater than LOP as evidenced by a more frequent occurrence of psoriasis amongst first degree family members in EOP when compared to LOP ${ }^{2}$. Although no data have been presented specifically in LOP, twin studies conducted to ascertain the proportion of heritability in the disease have included LOP patients and smaller scale genetic studies have reported associations indicating this subtype could also have a genetic component contributing to aetiology ${ }^{4}$. To date, studies involving LOP cohorts have failed to find an association with $H L A-C w 6$, the main susceptibility locus of EOP ${ }^{5 ; 6}$. More recently we have reported an association of IL1B with LOP, exclusive of EOP ${ }^{7}$. The two subtypes also have differing clinical presentations such as a more severe disease course in EOP versus LOP${ }^{2}$. Differences have also been noted in epidermal Langerhans' cell (LC) migration between the two subtypes ${ }^{8}$.

This combination of clinical, genetic and immunological observations indicates that whilst there is likely to be significant genetic overlap between these two phenotypes, there is the possibility that novel genetic loci may underpin some of the manifestations of LOP. 
This article is protected by copyright. All rights reserved.

The aim of this study was first, to investigate the genetic overlap between EOP and LOP and second, to identify loci that are specific to LOP. To do this we genotyped LOP samples using the Immunochip, a custom array designed to enable discovery, fine-mapping and replication studies of loci previously associated with autoimmune diseases ${ }^{9}$.

\section{Materials and Methods}

\section{Samples}

This study adheres to the Declaration of Helsinki Principles and was approved by the relevant local research ethics committees, and all subjects provided written informed consent.

Caucasian patients with late-onset (age of onset $\geq 40$ years) chronic plaque psoriasis were recruited to the study. Patients were recruited from the Dermatology Centre, Salford Royal NHS Foundation Trust at the University of Manchester; Guy's Hospital, London and the National Hospital for Rheumatic Diseases in Bath.

Data from healthy control samples were obtained from the 1958 British Birth Cohort and the UK Blood Service Collection as part of the Wellcome Trust Case-Control Consortium 2 project $^{10}$; (www.wtccc.org.uk).

\section{Genotyping}

All samples were genotyped using the Illumina Infinium Immunochip array according to manufacturer's protocols. This custom designed chip contains 196,524 single nucleotide polymorphisms (SNPs) from loci previously associated with 13 autoimmune diseases, including psoriasis. Its purpose is to help characterise the overlap in genetic susceptibility between these diseases and allows fine-mapping and replication of loci that have been previously identified in GWAS. It is the same platform used by Tsoi et al., $2012^{3}$ which allows direct comparison of the 36 
This article is protected by copyright. All rights reserved.

loci analysed in this paper. Genotype calling was implemented using Genotyping Module (v1.8.4) of the GenomeStudio Data Analysis software package.

\section{Quality Control}

A single project was created within GenomeStudio, with all genotyped samples included. Genotype clustering was performed using the GenTrain 2.0 clustering algorithm within GenomeStudio, incorporating Illumina's cluster file, Immunochip_Gentrain_June2010.egt and manifest file, Immuno_BeadChip_11419691_B.bpm. Initial sample quality control was carried out, excluding any with a call rate $<90 \%$. The remaining samples were then reclustered.

Markers were subjected to quality control measures using GenomeStudio's performance metrics. Markers were excluded based on cluster separation $<0.4$ and call rate $<98 \%$. Furthermore, any markers that were deemed unsuitable such as duplicates and monoallelic SNPs were removed. The GenomeStudio project was then exported for further downstream quality control and analysis.

For samples, identity by descent was carried out to identify related or duplicated samples according to outliers based on autosomal heterozygosity using the "--genome" function within PLINK (v1.07) ${ }^{11}$. If two or more samples were inferred to be related or duplicates, indicated by a Pi-HAT of $>0.15$, then the sample with the most amount of missing data was removed from the study. Principal components analysis (PCA) was carried out, merging the dataset with HapMap phase 2 samples containing European (CEU), Nigerian (YRI) and Chinese (CHB) reference populations to ensure all samples were of Caucasian ancestry and to prevent population stratification ${ }^{12}$. PCA was performed on a subset of SNPs, filtered for those in high correlation known as linkage disequilbrium (LD), and a minor allele frequency (MAF) $>5 \%$, using EIGENSOFT v4. $2^{13}$. 
This article is protected by copyright. All rights reserved.

\section{Imputation of HLA Gene Region}

Imputation analysis, a statistical process used to infer the identity of missing SNPs based on their correlation with SNPs directly genotyped in the dataset, was carried out for the HLA gene region using the SNP2HLA computational software and the Type 1 Diabetes Genetics Consortium dataset reference panel as previously described ${ }^{14}$.

\section{Statistical Analysis}

Statistical analysis was carried out using PLINK (v1.07). Any marker with a call rate $<98 \%$ in cases or controls, or a Hardy-Weinberg P-value $<1 \times 10^{-4}$ in controls were excluded from the dataset. SNPs with a minor allele frequency of $<5 \%$ were excluded given the study sample size and consequent power to detect association at low frequency SNPs. Samples were excluded based on a call rate $<98 \%$. Association analysis was carried out using an allelic model with Fisher's exact. Genotype frequencies in case samples were compared to those in control samples. In addition, stepwise logistic regression was carried out for the major histocompatibility complex $(\mathrm{MHC})$ region spanning $\sim 29-33 \mathrm{Mb}$ on chromosome 6, conditioning for the top hit in each step.

A correction for multiple testing was implemented using Genetic type 1 error calculator (GEC), which calculates the effective number of independent markers $\left(M_{e}\right)$ in the study, taking into account the extensive LD across the chip and produces a suggestive P-value threshold ${ }^{15}$.

\section{Confirming Specificity To Late-Onset Psoriasis}

Any novel regions identified in the LOP cohort were tested for association in the previously published WTCCC2 GWAS dataset, which is a predominantly EOP cohort of European ancestry ${ }^{16}$. This dataset consisted of 2178 samples and 5175 controls, with the locus of interest being analysed using an allelic model with Fisher's exact in PLINK (v1.07). 
This article is protected by copyright. All rights reserved.

\section{Functional Annotation}

All SNPs in high LD $\left(r^{2}>0.8\right)$ with novel markers were obtained using the Tabix and VCF tools softwares and data from the 1000 genomes project ${ }^{17-19}$. All SNPs were then interrogated for potential function using the ASSIMILATOR program and RegulomeDB database ${ }^{20 ; 21}$. SNPs were also analysed for possible association with differential expression of a gene, known as quantitative trait loci (eQTL). eQTL analysis was carried out using the Genevar and GTEx databases ${ }^{22 ; 23}$.

\section{Results}

A total of 543 Caucasian patients with LOP were recruited to the study. Data were available from 4,373 healthy controls. The mean age of onset of psoriasis was 51.1 years, standard deviation 9.0 years, range $40-81$ years. Males accounted for $54.1 \%$ and females $45.9 \%$ in the psoriasis cohort compared to $47.4 \%$ male and $52.6 \%$ female in the controls. After quality control (see Materials and Methods), 108,379 SNPs were suitable for analysis. A correction for multiple testing was implemented using the software tool Genetic type 1 error calculator (GEC; see Materials and Methods) which produced a threshold of $\mathrm{P}<2.30 \times 10^{-5}$ for claims of suggestive significance.

Nine loci were significantly associated with LOP at this threshold. Of these, seven loci have previously been associated with EOP in GWAS (Table 1). These include human leukocyte antigen C (HLA-C), interferon induced with helicase C domain 1 (IFIH1), interleukin-12B (IL12B), interleukin-23A (IL23A), interleukin-23R (IL23R), zinc finger protein 313 (ZNF313) and tumour necrosis factor receptor-associated factor 3 interacting protein 2 (TRAF3IP2). The strongest signals were from IL12B (rs2546890; $\mathrm{P}=7.16 \times 10^{-12}$, odds ratio $\left.(\mathrm{OR})=1.57\right)$ and $H L A-C\left(\mathrm{rs} 13191099 ; \mathrm{P}=3.73 \times 10^{-10}, \mathrm{OR}=\right.$ 1.72) for which the strength of association would be considered significant at genome-wide thresholds $\left(P=5 \times 10^{-8}\right)$. Interestingly the HLA-C SNP, rs13191099, is in moderate LD with HLA-Cw6 $\left(r^{2}=0.67\right)$, which itself reaches our study-wide significance threshold $\left(P=3.15 \times 10^{-6}, O R=1.60\right)$. 
This article is protected by copyright. All rights reserved.

Further investigation of the IL12B locus reveals that rs 2546890 lies in the exonic region of a long intergenic non-coding RNA (lincRNA) (LOC285626), whilst the index SNP for HLA-C (rs13191099) is intergenic between this gene and $H L A-B$.

The remaining loci, KIAA1919 in the TRAF3IP2 region (rs71562288; $\mathrm{P}=1.64 \times 10^{-6}$ ), IL23R $\left(\mathrm{rs72676067}, \mathrm{P}=1.87 \times 10^{-6}\right)$, SNAI1 in the ZNF313 region $\left(\mathrm{rs60813083} ; \mathrm{P}=5.53 \times 10^{-6}\right), I F I H 1$ $\left(\right.$ rs1990760; $\left.\mathrm{P}=7.97 \times 10^{-6}\right)$ and CNPY2 in the IL23A region $\left(r s 10876882 ; \mathrm{P}=8.11 \times 10^{-6}\right)$ showed suggestive evidence for association with LOP.

In addition to these EOP loci, there was an association that has not been identified before in a psoriasis GWAS (Table 2). This was at $\mathrm{rs} 887998\left(\mathrm{P}=8.81 \times 10^{-6}, \mathrm{OR}=1.40\right)$, an intronic variant in IL-1 receptor, type 1 (IL1R1), which maps to chromosome $2 \mathrm{q} 13$ and is a receptor molecule for cytokines IL1A, IL1 $\beta$ and IL1RA. There are eight SNPs that are in high LD with rs887998, all in intronic regions of IL1R1. In order to establish that the locus is specifically associated with LOP, we analysed 150 SNPs in the IL1R1/IL1R2 region in a published EOP GWAS cohort of 2,178 cases and 5,175 controls ${ }^{16}$. As expected, rs887998 was not associated with EOP ( $P=0.628$; Supplementary Table S1). The top most hit from the region was $r s 1108338(P=0.011)$, however this association is not significant when corrected for multiple testing and is not in high LD with rs887998 $\left(r^{2}<0.01\right)$.

As the MHC region is a well-known psoriasis susceptibility locus, with HLA-C particularly associated with the disease and high levels of LD often obscuring independent signals from other loci, imputation and stepwise logistic regression analysis was carried out for this region to identify associations that were independent of $H L A-C$. The analysis was conditioned on the top hit from the region (rs13191099) and then repeated for each subsequent top hit. From this one further locus was identified in the HLA-A region ( $r$ 22256919; $\mathrm{P}=2.54 \times 10^{-6}, \mathrm{OR}=1.38$ ); (Table 3).

In order to uncover how ILIR1 contributes to disease pathogenesis at a biological level, we carried out an eQTL analysis of the index SNP and all proxies using bioinformatic databases. Data was 
This article is protected by copyright. All rights reserved.

available for a range of tissues types including adipose, brain, liver, lymphoblastoid, monocytes and skin. Considering significant eQTL associations at $\mathrm{P}<1 \times 10^{-4}$, none of the SNPs from the IL1R1 region showed evidence of being eQTLs (Supplementary Table S2).

\section{Discussion}

To date, genetic association studies in psoriasis have focused almost exclusively on EOP patients. As a result, our knowledge of the underlying similarities and differences in the genetics between EOP and LOP is lacking. In this study we have demonstrated that seven loci previously associated with EOP in GWAS are significantly associated with LOP $^{24}$. One locus, HLA-A, has been associated with EOP in a smaller scale study and was identified in this study independently of $H L A-C$ after conditional analysis $^{25}$. Furthermore, we have identified a LOP specific locus at ILIR1 which has not previously been associated with psoriasis.

Interleukin-1R1 binds IL1 $\alpha$ and IL1 $\beta$. We have previously shown that SNPs rs16944 and rs11687624, in the $I L 1 B$ region were associated exclusively with $L^{7} P^{7}$. It is interesting to note that two SNPs from the IL1B region included on the Immunochip array (rs11676014 and rs1143633) showed similar association levels in this study $(P=0.02)$ when compared to the SNPs investigated in our previous study. Furthermore, rs11676014 is in high LD $\left(r^{2}=0.97\right)$ with rs11687624 from our previous study, with the discrepancy in P-values between studies potentially being attributable to a small difference in samples used. Linkage disequilibrium between the IL1B SNPs in both studies and the lead SNP from IL1R1 in this study ( $r s 887988)$ is small $\left(r^{2}<0.01\right)$, suggesting the two loci are independent of each other. IL1 $\beta$ and TNF- $\alpha$ are involved in epidermal LC migration to local draining lymph nodes, which is known to be differential between EOP and LOP. IL1 $\alpha$ and IL1 $\beta$ are also involved in regulation of the NF-KB pathway, along with IL36RN (IL1F5) which is a member of the IL1 family and has been associated with generalised pustular psoriasis ${ }^{26 ; 27} . I L 1 R 2$ has previously been associated 
This article is protected by copyright. All rights reserved.

with ankylosing spondylitis at GWAS significance levels ${ }^{28}$. It is interesting to note that the SNP identified in this study, rs887998, is located within an intronic region of an IL1R1 transcript. Initial bioinformatics using gene expression databases and gene annotation tools indicate that neither rs887998 nor nearby SNPs in high LD $\left(r^{2}>0.8\right)$ are likely to be eQTLs for IL1R1. However, expression data is not currently exhaustive for all cell types, particularly for skin. This is an obvious area for further functional investigation.

The association of EOP hits with LOP provide confirmation that the two subtypes follow similar pathogenesis. Furthermore, EOP and LOP may share common causative mutations as demonstrated by the presence of three SNPs that have been directly reported from GWAS. One of these, rs 2546890 in $I L 12 B$, has been directly genotyped in this dataset ${ }^{29}$. The other two, rs10484554 from HLA-C and rs2066808 from CNPY2 are in high LD with the lead SNP from their regions ${ }^{16 ; 30}$. It is interesting to note that the locus with the biggest effect size in EOP, HLA-C, is also associated with LOP despite previous studies suggesting otherwise ${ }^{5 ; 6}$. There are two possible explanations for this, the first is that the previous studies had small late-onset cohorts $(n=145)^{6}$ and therefore, had reduced power ( $27 \%$ power for an odds ratio of 1.5$)$ to detect an association compared to this study $(80 \% ; n=543)$. The second is that the age at onset of psoriasis that differentiates LOP from EOP is set too low at 40. Previous studies have attempted to counteract this by only including samples with age of psoriasis onset at 50 years or over ${ }^{6}$. However, most epidemiological studies would suggest 40 as a reasonable cut off with little overlap between an early and late cohort when looking at the bell curves. This approach is also consistent with our recent genetic investigations into $I L 1 B^{7}$. It is interesting to note the much smaller odds ratio at $H L A-C(O R=1.72)$ than that previously reported for EOP $(O R=4.32)^{3}$. This may imply that antigen presentation has a lesser role in LOP but the presence of an association at HLA-A indicates that more than one gene, relevant to antigen presentation, may contribute to LOP. 
This article is protected by copyright. All rights reserved.

Although only two of the loci identified in this study reached accepted levels for genome-wide significance $\left(P<5 \times 10^{-8}\right)$ it is likely this threshold is too stringent for the study design used. The markers that are on the Immunochip array were chosen due to prior evidence of association with one or more autoimmune disease. Thus the prior probability of finding an association in this study was increased. It has been proposed that genetic loci showing prior evidence of association need not have a conservative significance threshold due to the stringent nature in which they have been initially identified. One such example is shown in a study looking at shared loci between type 1 diabetes and celiac disease ${ }^{31}$, which considered significant associations at $\mathrm{P}<1 \times 10^{-4}$. Moreover, arrays containing large numbers of markers, such as the Immunochip, have blocks in high LD. This contradicts a key assumption of Bonferroni, the most popular method of correcting for multiple testing. Previous studies have overcome this problem by implementing a software tool, such as SNP spectral decomposition (SNPSpD) ${ }^{32}$, to calculate the number of independent SNPs $\left(M_{e}\right)$ and combine with a Bonferroni style correction, thereby producing a new significance threshold ${ }^{33}$. We used a similar method in this study with the GEC, which is a newer tool with an updated method of calculating $\mathrm{M}^{15}$.

Despite this, there are 29 loci from EOP GWAS that did not reach study-wide significance in this analysis (Supplementary Table S3). However 20 of these loci did show evidence of nominal association $(P<0.05)$. This does not necessarily represent non-involvement of these loci in LOP, but may reflect the smaller sample size $(n=543)$ compared to many GWAS ( $n=10,588$ for Tsoi et al., $2012)^{3}$. Future studies should therefore be carried out; first to replicate and validate the association of ILIR1 in an independent cohort, and second to provide a more comprehensive analysis of EOP loci in a larger LOP cohort.

In conclusion, we have demonstrated that LOP is a specific subtype of chronic plaque psoriasis that not only overlaps with EOP, but also has a unique set of genetic loci associated with it. These findings have the potential to benefit patients as the pathways associated with disease may identify targets 
This article is protected by copyright. All rights reserved.

for therapy development or repositioning. Future work should be focussed on pinpointing the causative mutations of these associations through further functional genomics.

\section{Acknowledgements}

H.L. Hébert is funded by a Ph.D. studentship from Abbott (now Abbvie);

J. Bowes and A. Barton are funded by Arthritis Research UK (grant ref. 20385).

R.B. Warren is an NIHR Clinical Senior Lecturer.

C.E.M. Griffiths is an NIHR Senior Investigator and is funded in part by the Medical Research Council and the NIHR Manchester Biomedical Research Centre.

\section{References}

1. Parisi R, Symmons DPM, Griffiths CEM et al. Global epidemiology of psoriasis: a systematic review of incidence and prevalence. J Invest Dermatol 2013; 133: 377-85.

2. Henseler T, Christophers E. Psoriasis of early and late onset: characterization of two types of psoriasis vulgaris. J Am Acad Dermatol 1985; 13: 450-6.

3. Tsoi LC, Spain SL, Knight J et al. Identification of 15 new psoriasis susceptibility loci highlights the role of innate immunity. Nat Genet 2012; 44: 1341-8.

4. Wongpiyabovorn J, Hirankarn N, Ruchusatsawat $\mathrm{K}$ et al. Association of the interleukin-10 distal promoter (-2763A/C) polymorphism with late-onset psoriasis. Clin Exp Dermatol 2008; 33: 1869.

5. Gudjonsson JE, Karason A, Runarsdottir EH et al. Distinct clinical differences between HLA$\mathrm{CW}^{*} 0602$ positive and negative psoriasis patients - an analysis of 1019 HLA-C- and HLA-B-typed patients. J Invest Dermatol 2006; 126: 740-5.

6. Allen $\mathrm{MH}$, Ameen $\mathrm{H}$, Veal $\mathrm{C}$ et al. The major psoriasis susceptibility locus PSORS1 is not a risk factor for late-onset psoriasis. J Invest Dermatol 2004; 124: 103-6.

7. Hébert HL, Bowes J, Smith RL et al. Polymorphisms in IL-1B Distinguish between Psoriasis of Early and Late Onset. J Invest Dermatol 2014; 134: 1459-62. 
This article is protected by copyright. All rights reserved.

8. Shaw FL, Cumberbatch M, Kleyn CE et al. Langerhans cell mobilization distinguishes between early-onset and late-onset psoriasis. J Invest Dermatol 2010; 130: 1940-2.

9. Cortes A, Brown M. Promise and pitfalls of the Immunochip. Arthritis Res Ther 2011; 13: 101.

10. Wellcome Trust Case Control Consortium. Genome-wide association study of 14,000 cases of seven common diseases and 3,000 shared controls. Nature 2007; 447: 661-78.

11. Purcell S, Neale B, Todd-Brown K et al. PLINK: a tool set for whole-genome association and population-based linkage analyses. Am J Hum Genet 2007; 81: 559-75.

12. The International HapMap Consortium. A second generation human haplotype map of over 3.1 million SNPs. Nature 2007; 449: 851-61.

13. Patterson N, Price AL, Reich D. Population structure and eigenanalysis. PLoS Genet 2006; 2: e190.

14. Jia $\mathrm{X}$, Han $\mathrm{B}$, Onengut-Gumuscu $\mathrm{S}$ et al. Imputing amino acid polymorphisms in human leukocyte antigens. PLOS ONE 2013; 8: e64683.

15. Li M, Yeung J, Cherny $S$ et al. Evaluating the effective numbers of independent tests and significant $p$-value thresholds in commercial genotyping arrays and public imputation reference datasets. Hum Genet 2011; 131: 747-56.

16. Strange A, Capon F, Spencer CC et al. A genome-wide association study identifies new psoriasis susceptibility loci and an interaction between HLA-C and ERAP1. Nat Genet 2010; 42: 985-90.

17. Abecasis $G$, Auton $A$, Brooks $L$ et al. An integrated map of genetic variation from 1,092 human genomes. Nature 2012; 491: 56-65.

18. Danecek $\mathrm{P}$, Auton A, Abecasis $\mathrm{G}$ et al. The variant call format and VCFtools. Bioinformatics 2011; 27: 2156-8.

19. Li H. Tabix: fast retrieval of sequence features from generic TAB-delimited files. Bioinformatics 2011; 27: 718-9.

20. Boyle AP, Hong EL, Hariharan $\mathrm{M}$ et al. Annotation of functional variation in personal genomes using RegulomeDB. Genome Res 2012; 22: 1790-7.

21. Martin P, Barton A, Eyre S. ASSIMILATOR: a new tool to inform selection of associated genetic variants for functional studies. Bioinformatics 2011; 27: 144-6.

22. Yang TP, Beazley C, Montgomery SB et al. Genevar: a database and Java application for the analysis and visualization of SNP-gene associations in eQTL studies. Bioinformatics 2010; 26: 2474-6.

23. Lonsdale J, Thomas J, Salvatore M et al. The Genotype-Tissue Expression (GTEx) project. Nat Genet 2013; 45: 580-5.

24. Hébert HL, Ali FR, Bowes J et al. Genetic susceptibility to psoriasis and psoriatic arthritis: implications for therapy. Br J Dermatol 2012; 166: 474-82. 
This article is protected by copyright. All rights reserved.

25. Knight J, Spain SL, Capon F et al. Conditional analysis identifies three novel major histocompatibility complex loci associated with psoriasis. Hum Mol Genet 2012; 21: 5185-92.

26. Sims JE, Smith DE. The IL-1 family: regulators of immunity. Nat Rev Immunol 2010; 10: 89-102.

27. Korber A, Mossner R, Renner R et al. Mutations in IL36RN in Patients with Generalized Pustular Psoriasis. J Invest Dermatol 2013; 133: 2634-7.

28. Reveille JD, Sims AM, Danoy P et al. Genome-wide association study of ankylosing spondylitis identifies non-MHC susceptibility loci. Nat Genet 2010; 42: 123-7.

29. Ellinghaus $E$, Ellinghaus $D$, Stuart PE et al. Genome-wide association study identifies a psoriasis susceptibility locus at TRAF3IP2. Nat Genet 2010; 42: 991-5.

30. Nair RP, Duffin KC, Helms $C$ et al. Genome-wide scan reveals association of psoriasis with IL-23 and NF-kB pathways. Nat Genet 2009; 41: 199-204.

31. Smyth DJ, Plagnol V, Walker NM et al. Shared and distinct genetic variants in type 1 diabetes and celiac disease. N Engl J Med 2008; 359: 2767-77.

32. Nyholt DR. A simple correction for multiple testing for single-nucleotide polymorphisms in linkage disequilibrium with each other. Am J Hum Genet 2004; 74: 765-9.

33. Fritsche LG, Chen W, Schu M et al. Seven new loci associated with age-related macular degeneration. Nat Genet 2013; 45: 433-9. 
This article is protected by copyright. All rights reserved.

Table 1 - Genotype counts of non-MHC SNPs associated with late-onset psoriasis from loci previously associated with early-onset psoriasis

in

\begin{tabular}{|c|c|c|c|c|c|c|c|c|c|c|c|c|c|c|}
\hline \multirow[b]{3}{*}{ SNP } & \multirow[b]{3}{*}{ Chr } & \multirow{3}{*}{$\begin{array}{c}\text { Locus } \\
\text { (nearest gene) }\end{array}$} & \multirow{3}{*}{$\begin{array}{c}\text { Risk/ } \\
\text { Non-risk } \\
\text { Allele }\end{array}$} & \multirow{3}{*}{$\begin{array}{c}\text { RAF } \\
\text { (cases) }\end{array}$} & \multirow{2}{*}{\multicolumn{3}{|c|}{$\begin{array}{c}\text { Cases }(n=543) \\
\text { Genotype Frequency }\end{array}$}} & \multirow{2}{*}{\multicolumn{3}{|c|}{$\begin{array}{l}\text { Controls }(\mathrm{n}=4,373) \\
\text { Genotype Frequency }\end{array}$}} & \multirow{3}{*}{$\begin{array}{c}\text { RAF } \\
\text { (controls) }\end{array}$} & \multirow[b]{3}{*}{ Allelic P } & \multirow[b]{3}{*}{ OR } & \multirow[b]{3}{*}{$\mathrm{Cl}_{95 \%}$} \\
\hline & & & & & & & & & & & & & & \\
\hline & & & & & 11 & 12 & 22 & 11 & 12 & 22 & & & & \\
\hline rs2546890 & 5 & $I L 12 B$ & $A / G$ & 0.625 & 206 & 265 & 71 & 1159 & 2180 & 1030 & 0.515 & $7.16 \times 10^{-12}$ & 1.57 & $1.38-1.79$ \\
\hline rs13191099 & 6 & $H L A-C$ & $\mathrm{G} / \mathrm{A}$ & 0.195 & 21 & 170 & 352 & 65 & 952 & 3356 & 0.124 & $3.73 \times 10^{-10}$ & 1.72 & $1.46-2.02$ \\
\hline rs71562288 & 6 & $\begin{array}{c}\text { TRAF3IP2 } \\
\text { (KIAA1919) }\end{array}$ & $\mathrm{G} / \mathrm{A}$ & 0.137 & 14 & 121 & 408 & 36 & 713 & 3624 & 0.090 & $1.64 \times 10^{-6}$ & 1.61 & $1.34-1.95$ \\
\hline rs72676067 & 1 & IL23R & $A / G$ & 0.376 & 73 & 262 & 208 & 418 & 1815 & 2136 & 0.303 & $1.87 \times 10^{-6}$ & 1.38 & $1.21-1.58$ \\
\hline rs60813083 & 20 & ZNF313 & $C / A$ & 0.116 & 9 & 108 & 426 & 24 & 604 & 3740 & 0.075 & $5.53 \times 10^{-6}$ & 1.63 & $1.33-1.99$ \\
\hline rs1990760 & 2 & $|F| H 1$ & $A / G$ & 0.676 & 250 & 234 & 59 & 1611 & 2080 & 682 & 0.606 & $7.97 \times 10^{-6}$ & 1.35 & $1.18-1.55$ \\
\hline rs10876882 & 12 & $\begin{array}{c}\text { IL23A } \\
\text { (CNPY2) }\end{array}$ & $\mathrm{G} / \mathrm{A}$ & 0.963 & 504 & 38 & 1 & 3780 & 563 & 27 & 0.929 & $8.11 \times 10^{-6}$ & 1.99 & $1.43-2.75$ \\
\hline
\end{tabular}

\section{GWAS}

SNP, single nucleotide polymorphism; Chr, chromosome; RAF, risk allele frequency; OR, odds ratio; $\mathrm{Cl}$, confidence interval.

$1=$ Minor Allele

2 = Major Allele 
This article is protected by copyright. All rights reserved.

Table 2 - Genotype counts of SNPs associated with late-onset psoriasis, not previously identified with any type of psoriasis SNP, single nucleotide polymorphism; Chr, chromosome; RAF, risk allele frequency; OR, odds ratio; $\mathrm{Cl}$, confidence interval.

\begin{tabular}{|c|c|c|c|c|c|c|c|c|c|c|c|c|c|c|}
\hline \multirow[b]{3}{*}{ SNP } & \multirow[b]{3}{*}{$\mathrm{Chr}$} & \multirow[b]{3}{*}{ Gene } & \multirow{3}{*}{$\begin{array}{l}\text { Risk Allele/ } \\
\text { Non-risk } \\
\text { Allele }\end{array}$} & \multirow{3}{*}{$\begin{array}{c}\text { RAF } \\
\text { (cases) }\end{array}$} & \multirow{2}{*}{\multicolumn{3}{|c|}{$\begin{array}{c}\text { Cases }(n=543) \\
\text { Genotype Frequency }\end{array}$}} & \multirow{2}{*}{\multicolumn{3}{|c|}{$\begin{array}{l}\text { Controls }(n=4,373) \\
\text { Genotype Frequency }\end{array}$}} & \multirow{3}{*}{$\begin{array}{c}\text { RAF } \\
\text { (controls) }\end{array}$} & \multirow[b]{3}{*}{ Allelic P } & \multirow[b]{3}{*}{ OR } & \multirow[b]{3}{*}{$\mathrm{Cl}_{95 \%}$} \\
\hline & & & & & & & & & & & & & & \\
\hline & & & & & 11 & 12 & 22 & 11 & 12 & 22 & & & & \\
\hline rs887998 & 2 & IL1R1 & $\mathrm{A} / \mathrm{G}$ & 0.262 & 32 & 220 & 291 & 189 & 1387 & 2797 & 0.202 & $8.81 \times 10^{-6}$ & 1.40 & $1.21-1.62$ \\
\hline
\end{tabular}

$1=$ Minor Allele

2 = Major Allele

\begin{tabular}{|c|c|c|c|c|c|c|c|c|c|c|c|c|c|}
\hline \multirow[b]{3}{*}{ SNP } & \multirow[b]{3}{*}{$\mathrm{Chr}$} & \multirow[b]{3}{*}{ Gene } & \multirow{3}{*}{$\begin{array}{c}\text { Risk } \\
\text { Allele } \\
\end{array}$} & \multirow{2}{*}{\multicolumn{3}{|c|}{$\begin{array}{c}\text { Cases }(n=543) \\
\text { Genotype Frequency }\end{array}$}} & \multirow{2}{*}{\multicolumn{3}{|c|}{$\begin{array}{l}\text { Controls }(n=4,373) \\
\text { Genotype Frequency }\end{array}$}} & \multirow{3}{*}{$\begin{array}{l}\text { Non- } \\
\text { Risk } \\
\text { Allele }\end{array}$} & \multirow[b]{3}{*}{ Allelic P } & \multirow[b]{3}{*}{ OR } & \multirow[b]{3}{*}{$\mathrm{Cl}_{95 \%}$} \\
\hline & & & & & & & & & & & & & \\
\hline & & & & 11 & 12 & 22 & 11 & 12 & 22 & & & & \\
\hline rs2256919 & 6 & $H L A-A$ & $A$ & 250 & 242 & 50 & 1612 & 2031 & 727 & C & $2.54 \times 10^{-6}$ & 1.38 & $1.21-1.58$ \\
\hline
\end{tabular}

Table 3 - Genotype counts of SNPs associated with late-onset psoriasis from the MHC region

SNP, single nucleotide polymorphism; Chr, chromosome; bp, base position; OR, odds ratio; $\mathrm{Cl}$, confidence interval.

$1=$ Minor Allele

2 = Major Allele 